\title{
Come the revolution
}

\author{
REVISITING... THE MANAGEMENT OF ANOREXIA NERVOSA
}

\section{Bob Palmer}

Abstract Over the past decade there have been few advances and no real breakthroughs in the treatment and management of anorexia nervosa. There has perhaps been a consolidation of opinion and practice. A clinical guideline for eating disorders published under the auspices of the National Institute for Clinical Excellence (NICE) has summarised our present ideas of good practice as well as highlighting the lack of good evidence on which to base this.

Bob Palmer revisits a topic on which he first wrote for APT 10 years ago (Palmer, 1996). His 1996 article is available on our website (http://apt.rcpsych.org), as a data supplement to the online version of the present submission.

It would be good to think that there were major advances to report in the management of anorexia nervosa in the 10 years since I first wrote on the subject for APT (Palmer, 1996). However, I fear that this is not the case. The field moves only slowly. Thus, the present account will not only cover much the same ground as the earlier piece, but could in principle reproduce some of the sections without alteration. However, most have been changed to reflect a different perspective if not a new scene. The two articles are in some ways complementary. As befits the times, the present article will make more reference to government and other documents.

It is now about 130 years since anorexia nervosa was first clearly described. Charles Lasègue and William Gull both contributed clinical descriptions at about the same time in the 1870s. Arguably it was the Frenchman who gave the richer account of the disorder but it was the Englishman who coined the term that would last, anorexia nervosa. It is perhaps 40 or so years since the truly modern concept of the disorder came to be established through the work of such authors as Hilde Bruch, Arthur Crisp and Gerald Russell. And still after all this time, the management and treatment of the disorder remain unsatisfactory in most respects. Some people still die of it (Sullivan, 1995). Many more have their youth and sometimes their whole lives blighted by it and yet there is no standard treatment of demonstrated efficacy. Furthermore, there is a relatively small amount of research into the management of anorexia nervosa compared with that for its sister disorder, bulimia nervosa, or even for the new and provisional condition, binge eating disorder. So, although it would be good to think that a major advance in treatment is just around the corner, such optimism would be somewhat cockeyed. Likewise, it would be good to think that what is generally considered to be good practice in the management of the disorder is readily available throughout the UK, but that too would be an unduly rosy view. Many new services are being developed but provision is still patchy (Royal College of Psychiatrists, 2000). We continue to do rather poorly in our attempts to help people with this serious and sometimes vicious disorder.

Arguably, in the past the poor response to the eating disorders, including anorexia nervosa, arose because they were not considered to be important mental disorders. Too often they were thought to be trivial, as something to do with silly young women and fashion, and as such not worthy of much attention. However, there have been signs recently that this image problem is being overcome. First, an influential paper listed eating disorders as being among the most lethal of functional mental disorders in the UK (Harris \& Barraclough, 1998). Second, the National Service Framework for Mental Health recognised their significance, recommending that 'individuals with severe disorders should be referred for specialist assessment, including a full medical and psychiatric assessment' (Department of Health, 1999: p. 30). Third and arguably most importantly, the National Institute for Clinical Excellence (now the National Institute for Health

Bob Palmer is a senior lecturer in psychiatry at University of Leicester Medical School (University of Leicester School of Medicine, Division of Psychiatry, Department of Health Sciences, Brandon Mental Health Unit, Leicester General Hospital, Gwendolen Road, Leicester LE5 4PW, UK. Tel.: 0116225 6286; e-mail: rlp@leicester.ac.uk) and an honorary consultant psychiatrist with Leicestershire Partnership NHS Trust. He has published on the subject of eating disorders and is Editor of the European Eating Disorders Review. 
and Clinical Excellence; NICE) chose eating disorders as one of the first topics for which clinical guidelines should be developed. A group under the chairmanship of Professor Simon Gowers produced National Clinical Guideline Number CG9, which was published early in 2004 (National Collaborating Centre for Mental Health, 2004; Wilson \& Shafran, 2005). This is available in a number of forms, including a summary version and a version designed for the general public.

The NICE group encountered the dearth of good evidence about anorexia nervosa mentioned above (Fairburn, 2005). The standard method, adopted by NICE, is for evidence to be classified according to a hierarchy, with an A rating being given to recommendations based on sound evidence from adequate randomised controlled trials and lower ratings being given for lesser evidence. However, none of the 48 specific recommendations about the management of anorexia nervosa received an $\mathrm{A}$ rating and almost all were given a $C$ because they were based solely on 'reports or opinions and/or clinical experiences of respected authorities' (National Collaborating Centre for Mental Health, 2004: p. 24). Nevertheless, the NICE guideline is an important development. It does represent a good summary of present best practice in treatment and management. The following account refers to many of its key recommendations.

\section{Diagnosis and assessment}

Diagnosis is the start of management. However, the recognition of anorexia nervosa is usually not difficult. Indeed, it is one of the few psychiatric conditions that may be guessed at in the street or across a crowded room. Most young women who look as though they have anorexia nervosa probably do. The diagnostic criteria in ICD-10 (World Health Organization, 1992) and DSM-IV (American Psychiatric Association, 1994) have not been revised in the past decade. The two systems are similar, although ICD-10 assigns people with low weight and bingeing and purging to the diagnosis of bulimia nervosa, whereas DSM-IV assigns them to a subtype of anorexia nervosa. In this respect, DSM-IV is in line with the intuitions of most clinicians and is perhaps superior. In just a few cases important diagnostic doubt may remain even after a thorough initial assessment. In such rare instances, it is important for the psychiatrist or other diagnosing professional to preserve uncertainty, especially with regard to the possibility of a hidden physical cause for weight loss. Anorexia nervosa should not be a diagnosis of exclusion, which is made when physicianly investigation draws a blank. Sometimes the psychopathology is atypical but it should be there. The diagnosis should be made on positive grounds.

Once an eating disorder is suspected the clinician will need to make particular enquiries about weight and eating over and above the usual questions that contribute towards a psychiatric diagnosis. However, the general mental state examination is important too, since many people presenting with anorexia nervosa will have depression, anxiety or other symptoms, or even full comorbid syndromes (Bulik, 2002). Likewise, risk of suicide and self-harm should also be addressed.

Some assessment of the person's physical state is necessary. Is the patient at significant physical risk because of her disorder? (Throughout this article, I shall refer to the patient as being female. Most are and it avoids the awkward 'he or she' or 'they'.) As a general rule it would be usual to perform a full blood count, urea, electrolyte and liver function tests on all patients with significant weight loss, and an electrocardiogram on most. Thyroid function tests are often done to exclude another cause of weight loss with anxiety, but in practice the differential diagnosis with hyperthyroidism should usually be evident on clinical grounds. Indeed, in general, clinicians should not neglect to use their experience and clinical nous. Does the patient look very ill? What is she able to do? Does she have physical symptoms? The squat test - asking the patient to squat and then rise without assistance - is useful to assess myopathy, and failing it is an indicator that the individual's body is losing the ability to compensate for malnourishment. Weight or body mass index (BMI: weight in kilograms divided by height in metres squared) alone is an inadequate index of risk. The trajectory of weight loss is relevant. Thus, a patient with a BMI of 12 who has been at the same weight for many months may be at less risk than another with a BMI of 14 who has lost $5 \mathrm{~kg}$ in the past month. Likewise, the implications of major weight loss may be more serious in a 13-yearold than in a 30-year-old. A full discussion of physical risk assessment is beyond the scope of this article but there are useful accounts in the NICE guideline CG9 and in a recent College document (Royal College of Psychiatrists, 2005).

And, of course, it is necessary to weigh the patient or to seek to do so. Some individuals will be reluctant to be weighed. Refusal faces the clinician with a real dilemma. Is it better to push the matter and run the risk of seeming to be the one who is making weight 'too important'? Or is going along with not weighing her missing out on essential information and unhelpfully endorsing a position of fearful avoidance? Handled badly, this dilemma can be the start of a battle with the patient. Handled 
well, it can be an opportunity to explore the patient's feelings and fears about weight, together with the dilemmas and difficulties that await her on any pathway out of the disorder. With such 'talking to death' of the issue, it is unusual for a patient to continue to refuse to be weighed. For adults, calculation of the BMI is useful. For children under 16 , reference to percentile tables is preferable.

There is much more to the assessment of someone with anorexia nervosa than just making a descriptive diagnosis. Two further issues are important and both relate to the meaning of the experience of the disorder. The first is the issue of engagement with the patient. The second is the need to attempt to understand why this person has fallen ill and why she has stayed trapped within the condition.

\section{Engagement}

During an initial encounter with a patient with anorexia nervosa, it is important to remember that the patient is assessing the assessor as well as the other way around. Does the clinician seem to be knowledgeable, confident and trustworthy? Does the patient feel that the clinician is listening? On the answer to these questions will depend whether the patient feels safer or more threatened at the end of the first encounter and hence more or less likely to be willing to think about change or even to come along for a second appointment.

It is a fair assumption that the patient will come along to an initial assessment with profoundly mixed feelings. Of course, she will not always be forthcoming about them. The notion of mixed feelings is different from the often-mentioned idea that the typical person with anorexia nervosa is inherently resistant to change. This is sometimes even described as a part of the illness. However, it seems more accurate to say that the person dislikes the state that she is in but also fears changing and moving out of it. The potential patient may feel between the devil and the deep blue sea. However, if the clinician pushes too strongly the case for change then the patient is likely to express only the case against. She will appear to simply oppose. Unproductive or even dangerous battling may ensue. Rather, the clinician's task is to give the patient opportunity to express both sides of the dilemma. The sooner this is done the better. Indeed, a good opening question in a first assessment interview is to ask the patient what she feels about coming along and then to actively facilitate exploration of the full range of thoughts and emotions. If the patient insists that she has only negative feelings about the consultation, it may be useful to give her the choice to opt out of the interview. In practice patients hardly ever do. In my experience of several hundred assessments, only one patient has ever actually walked out and she subsequently returned. However, having the option of leaving allows the patient to express some positive commitment to the process of assessment. Time spent in bringing out the mixed feelings and nurturing any positive wish to change is time well spent even at the very beginning. It is the start of the process of engagement and ideally the start of a therapeutic relationship that will sustain the patient throughout her journey towards recovery.

\section{The story}

The patient's attitude to assessment and to potential change will depend on the meaning for her of the experience of anorexia nervosa. Therefore another task of assessment, apart from diagnosis and risk assessment, is to begin to explore such meaning. The clinician needs to think about possible answers to the question 'why has this patient developed anorexia nervosa and why has she not recovered?' There is a need to try to tell a 'story' or indeed several stories about the patient. Such stories will arise through an attempt at empathetic understanding of the patient's emotions, motives and intentions. The task is to speculate about the origins of the disorder from the patient's perspective and about how she experiences it now. What is it like to be her?

I prefer the term 'story' to anything more highfaluting because it is important that any such account should always be thought of as fallible and provisional. A story that is wrong and based on too confident a jump from the patient's account, or worse still is derived directly from some general conviction or theorising, may get in the way. It could even jeopardise the all-important relationship with the patient. A good story, however, can be an invaluable aid to the clinician's attempt to engage the patient in considering and then making change.

The understanding of the patient's situation may be importantly enhanced by the inclusion of significant others in the process of assessment. Talking with the family of origin may be especially relevant. Indeed, almost all child and adolescent psychiatrists and many adult psychiatrists would do so routinely. The available stories of the antecedents and origin of the disorder are likely to be importantly enriched by including elements derived from the parental perspective. One leading authority, Arthur Crisp, sees anorexia nervosa as psychobiological retreat in the face of difficulties for the patient and her family in meeting the developmental challenges of adolescence broadly defined and suggests that the family should always be involved in the assessment 
process, regardless of the age of the patient (Crisp, 1980). However, this may not always be possible. Furthermore, patients on occasion veto such involvement and if they are adults it is important that they should be able to do so. Indeed, the NICE guideline CG9 emphasises that even younger patients should have the opportunity at some point in the assessment to talk with the assessing clinician without their parents being present.

\section{The tasks of recovery}

Ideally, assessment should conclude with a diagnosis made and the patient engaged in the venture of trying to understand her disorder and thinking about how to change. She may still have mixed feelings - indeed, if she does not acknowledge these she may well be being less than open - but she should be willing to continue contact and to think about leaving anorexia nervosa behind. The clinician needs to find some common language with the patient in which to frame the dilemmas and tasks ahead.

It may be useful to divide the tasks of recovery into three (Box 1). The first is easy to talk about but may be the most difficult to face for the patient; it is the restoration of a healthy weight and a style of eating that will sustain this. The second and third are not only difficult to do but they are also not easy to define. The second is the separation of ideas about weight and eating from the wider personal issues and meanings with which they have become entangled. This is essentially the dismantling of the so-called specific psychopathology of the disorder. The third is getting life on the move again and perhaps finding new and more adaptive ways of dealing with any issues that contributed to the patient's falling ill in the first place, together with any consequences of having been ill.

Thus, a woman who is now aged 26 and who fell into anorexia nervosa at 16 in the midst of an adolescence that had been complicated by the effects of sexual abuse at age 6 will need to find ways of negotiating both the challenges of a rekindled adolescence and the fact that her life has been

\section{Box 1 The tasks of recovery}

- Restoration of a healthy weight and a style of eating that will sustain it

- Separation of ideas about weight and eating from the wider personal issues and meanings with which they have become entangled

- Getting life on the move again distorted by the disorder for a decade. In some ways, through her suffering, she may feel older than her peers, but in other ways she may feel very young and ill equipped for many developmental hurdles that most people of her age successfully jumped years before.

\section{The stages of change}

Over recent years, the idea of stages of change - or rather of readiness for change - has been widely invoked in relation to anorexia nervosa (Treasure \& Bauer, 2005). It is rightly pointed out that the offer of treatments and interventions needs to be in tune with the patient's attitude to their situation. Thus, if the person is in so-called pre-contemplation then they may need to be helped to weigh up the pros and cons of change rather than having the means to change prematurely foisted on them. Help to change needs to be provided for those who have moved on through 'contemplation' and into 'action'. The techniques of motivational interviewing, pioneered in the world of substance misuse, may be used to try to promote such progress. ${ }^{1}$ Clearly, such ideas contain an important perspective on helping people with anorexia nervosa. However, the actual complexity of the mixed feelings experienced by many with the disorder may swamp even the relative sophistication of this model. The mixed feelings of someone with anorexia can be very mixed indeed and there is no real substitute for exploring these on an individual basis.

\section{The aspects of response}

If there are three aspects of recovery, it may be useful to think of our responses too as involving at least two and arguably three separable parts (Palmer \& Treasure, 1999). First, there is what might be called 'therapy', which is the personal contact between an individual patient and an individual clinician or perhaps a team designed to promote psychological change. Second, there is 'management', by which is meant the wider interventions, such as hospital admission or day programme attendance, aimed at providing a circumstance in which the patient may find it easier to change. And last, an appropriate 'service organisation' is required to provide both in appropriate combinations. These distinctions would seem to be unduely pedantry were it not for the fact

1. This topic has been discussed in an earlier issue of $A P T$ : see Treasure, J. (2004) Motivational interviewing. Advances in Psychiatric Treatment, 10, 331-337. Ed. 


\section{Box 2 Pharmacological treatment}

'Medication should not be used as the sole or primary treatment for anorexia nervosa'

(National Collaborating Centre

for Mental Health, 2004: p. 94)

'I would rather trust to moral influences and feeding than to medicines, though these might be still amongst the adjuvantia'

(Gull, 1874)

that the different aspects of response to anorexia nervosa do seem at times to be muddled. Each of the aspects of our response to the disorder will now be discussed separately although of course in practice they overlap.

\section{Therapy}

No drugs have been shown to be of specific benefit in the treatment of anorexia nervosa. The chief treatment must therefore be psychological in nature (Box 2).

Unfortunately, no one approach has been demonstrated to be convincingly better than another (Fairburn, 2005). This leaves experienced therapists free to follow their opinions and preferences, and the uncommitted unsure what is best. Nevertheless, there is some measure of consensus about the nature of good therapy for anorexia nervosa (Box 3).

The treatment needs to be prolonged. Furthermore, it needs to continue to address the mixed feelings about change that are likely to remain well past the initial engagement phase. Even more than in most therapies, helping the patient to feel safe and contained is central. Thus, issues such as reliability, consistency, empathy and clear boundaries need to be cherished. That having been said, the therapist needs to be flexible and willing to attend to the physical as well as the psychological issues presented by the patient. (This holds true even when another clinician is undertaking the main role of physical monitoring.) Therapists from the psychodynamic tradition may need to be more active than usual, whereas those with a cognitivebehavioural orientation may need to spend more time than usual exploring the complexities of their patients' attitudes to their illness. Thus, the former may need to be thinking uncharacteristically about the details of their patient's diet and perhaps using techniques such as food diaries, whereas the latter may find themselves pondering the complex ways in which the patient experiences their therapeutic suggestions and the therapeutic relationship within which they are offered.

\section{Box 3 NICE on out-patient treatment}

- Most people with anorexia nervosa should be treated on an out-patient basis

- Therapies to be considered for the psychological treatment of the disorder include cognitive analytic therapy (CAT), cognitivebehavioural therapy (CBT), interpersonal psychotherapy (IPT), focal psychodynamic therapy and family interventions focused explicitly on eating disorders

- Out-patient management should involve a psychological treatment (with physical monitoring) provided by a healthcare professional competent to give it and to assess the physical risk of the illness to the patient

- Out-patient psychological treatment and physical monitoring should normally continue for at least 6 months

- If there is significant deterioration during out-patient psychological treatment or there has been no significant improvement by the end of an adequate course of such treatment, more intensive forms of treatment should be considered

National Collaborating Centre for Mental Health (2004)

\section{Box 4 NICE on children and adolescents}

- Children and adolescents with anorexia nervosa should be offered family interventions that directly address the eating disorder

- In the treatment of children and adolescents it is particulary important that any in-patient management be provided within reasonable travelling distance to enable relatives and carers to be involved in the treatment, to maintain social and occupational links and to avoid difficulty in transition between primary and secondary care services

- Admission should be to age-appropriate facilities (with the potential for separate child and adolescent services) that have the capacity to provide appropriate educational and related activities

National Collaborating Centre for Mental Health (2004)

For adolescent patients, there is near consensus that it is helpful for clinicians to involve the family in treatment (Box 4). There is some evidence to back this up (Russell et al, 1987). However, the evidence 
suggests that the nature of this involvement may equally well be conjoint family therapy (patient, family and therapist meeting together) or family counselling in which the clinician meets separately with the patient and her family (Eisler et al, 2005). Furthermore, there is considerable enthusiasm at present for multifamily approaches in which perhaps half a dozen families meet together in intensive sessions that characteristically occur over several days (Dare \& Eisler, 2002). The evidence that family approaches are superior to individual work for younger patients is limited and arises mainly from the work of expert and enthusiastic therapists. It would probably be an error to infer that any family approach is always superior to any individual therapy.

\section{Management}

Most people presenting with anorexia nervosa can and should be treated in an out-patient setting. Indeed, except for people who present in extreme physical states, in-patient treatment should generally be reserved for those who have failed to progress with appropriate out-patient therapy (Box 5). In practice, the better the out-patient therapy the less the need for admission. However, a significant minority of patients - perhaps $10-20 \%$ in most places - require admission at some stage of their illness. Some require relatively brief admission to a medical ward for intervention for their physical state. However, admission for treatment of the disorder itself is a more major undertaking. Such admissions are almost always to a mental health facility and are often prolonged over several months or longer. Day care or intensive home treatment have been explored as an alternative to full admission. Their place has yet to be clarified (Birchall et al, 2002; Robinson, 2003). Indeed, as with the nature of the therapy, there is no satisfactory evidence for the efficacy of in-patient treatment itself (Crisp et al, 1991; Meads et al, 2001). Nevertheless, few experienced clinicians would deny the value of such treatment for some patients.

Admission is perhaps best thought of not as something done to the patient but as the provision of additional means which she may use in her own struggle towards recovery. Such treatment should usually be carried out in a unit where staff have experience of dealing with anorexia, usually therefore within a specialist centre. However, hospitalisation - even of the most expert kind hardly ever constitutes a full response to anorexia nervosa. There is a need for continuing therapy after admission. Ideally, therapy would continue without a break before, during and after admission. However, service organisation may often not allow this.

\section{Box 5 NICE on in-patient treatment}

- In-patient or day patient treatment should be considered if the disorder does not improve with appropriate out-patient treatment, or if there is a significant risk of suicide or severe self-harm

- If in-patient treatment is required, patients should be admitted to a setting in which skilled refeeding and careful physical monitoring (particularly for the first few days of refeeding) are available in combination with psychosocial interventions

- In-patients should follow a structured symptom-focused treatment regimen with the expectation of weight gain to achieve weight restoration. It is important to carefully monitor the patient's physical status during refeeding

- In-patients should receive psychological treatment that focuses on eating behaviour and attitudes to weight and shape, and also on wider psychosocial issues; again weight gain should be expected

- Following in-patient weight restoration the individual should be offered out-patient psychological treatment with the same focuses as those held during hospitalisation (eating behaviour, attitudes to weight and shape, and wider psychosocial issues). Both physical and psychological risk should be monitored regularly. Typically, this outpatient treatment and physical monitoring following in-patient weight restoration should continue for at least 12 months

National Collaborating Centre for Mental Health (2004)

\section{Service organisation}

Until recent years, specialist treatment for anorexia nervosa has largely huddled within and around prestigious centres with in-patient facilities. Over the past decade, many new local services have been created, usually without beds of their own. An important motivation for such developments has been a more equitable and efficient use of resources or even an attempt to save money in absolute terms. In general, the new services have had some success in these terms, perhaps by better selection of those few people who still need in-patient care. However, where the relationships between the local services and the in-patient units to which their patients may be admitted remain haphazard and ad hoc - as they often do - the desirable continuity of care remains 
an aspiration. Too often the patient's career still resembles a disorganised game of pass the parcel as she is moved from one service to another. How to overcome this remains a key issue of service provision. Some sort of managed clinical network or hub and spoke system where a group of local services share an in-patient facility is probably the best resolution for the issue and some systems of this type are being developed (Palmer \& Treasure, 1999). Such confederations of services could also share frameworks for clinical governance, training, supervision and research.

\section{Exceptional cases}

The old legal saying goes that hard cases make bad law. The same applies in the treatment of anorexia nervosa. Thus, a relatively inexperienced clinician may tend to generalise from the risks and intractability of the few most severe cases. For instance, a psychiatrist who sees an occasional patient with anorexia nervosa may use compulsion under the Mental Health Act 1983 much more than the specialist who sees many cases. The law is clear. Anorexia is a mental illness for which feeding is treatment. But most specialists would see compulsion as a last resort that is used, if at all, only after skilful attempts to work with the patient's mixed feelings have failed and the risks remain very high. My own view is that the treatment of anorexia nervosa under conditions of compulsion is a very specialised business - on a par with heart transplant surgery - and should be restricted to just a few centres.

Other 'hard cases' include individuals with major DSM-IV Axis I or II comorbidity. Unfortunately, in the presence of good out-patient services, the people who are admitted to hospital tend to include a disproportionate number with severe affective disorder, substance misuse or suicidal and selfharming behaviours. They can have major effects on the ethos of an in-patient unit. The treatment of severe anorexia nervosa - requiring the cooperation of the patient - in the presence of significant selfharming behaviour or suicidal intent arising through major depression and/or borderline personality disorder is complex and demanding. The use of particular therapies such as dialectical behaviour therapy may be helpful, but if anyone has a really good response to such situations I would be grateful if they would tell me (Palmer et al, 2003).

Lastly, people with truly chronic anorexia nervosa present challenges for which there are no easy answers. It would seem appropriate to strike a balance, keeping hope of recovery alive - the evidence would seem to support the thought that recovery is never impossible - but avoiding persecuting the individual with repeatedly failing attempts at treatment. Keeping in touch would seem to be important, as is the monitoring of physical issues and risks, such as that of osteoporosis, although the remedy in that case is also not especially clear.

\section{Conclusions}

The past decade has brought only minor evolution and certainly no revolution to the management of anorexia nervosa (Palmer, 2000). If in another 10 years someone were to be once again addressing the task of reviewing progress, I hope that they would be able to report both some substantial advances in treatment - a psychological treatment with demonstrably major benefits and perhaps a drug with some specific effect - and a more rational pattern of services. The first will require ingenuity and good research, the second a collective will. Work has to be done now if we are to achieve these goals by then.

\section{Declaration of interest}

None.

\section{References}

American Psychiatric Association (1994) Diagnostic and Statistical Manual of Mental Disorders (4th edn) (DSM-IV). Washington, DC: APA.

Birchall, H., Palmer, R. L., Waine, J., et al (2002) Intensive day programme treatment for severe anorexia nervosa - the Leicester experience. Psychiatric Bulletin, 26, 334-336.

Bulik, C. M. (2002) Anxiety, depression and eating disorders. In Eating Disorders and Obesity: A Comprehensive Handbook (2nd edn) (eds C. G. Fairburn \& K. D. Brownell). New York \& London: Guilford Press.

Crisp, A. H. (1980) Anorexia Nervosa: Let Me Be. London: Academic Press.

Crisp, A. H., Norton, K., Gowers, S., et al (1991) A controlled study of the effect of therapies aimed at adolescent and family psychopathology in anorexia nervosa. British Journal of Psychiatry, 159, 325-333.

Dare, C. \& Eisler, I. (2002) A multi-family group day treatment programme for adolescent eating disorder. European Eating Disorders Review, 8, 4-18.

Department of Health (1999) National Service Framework for Mental Health. Modern Standards and Service Models. London: Department of Health.

Eisler, I., le Grange, D. \& Asen, E. (2005) Family interventions. In The Essential Handbook of Eating Disorders (eds J. Treasure, U. Schmidt \& E. van Furth). Chichester: John Wiley \& Sons.

Fairburn, C. G. (2005) Evidence-based treatment of anorexia nervosa. International Journal of Eating Disorders, 37 (suppl. 1), S26-30.

Gull, W. (1874) Anorexia nervosa (apepsia hysterica, anorexia hysterica). Transactions of the Clinical Society of London, 7, 222-228.

Harris, E. C. \& Barraclough, B. (1998) Excess mortality of mental disorder. British Journal of Psychiatry, 173, 11-53.

Meads, C., Gold, L. \& Burls, A. (2001) How effective is out-patient care compared to in-patient care for the treatment of anorexia nervosa? A systematic review. European Eating Disorders Review, 9, 229-241.

National Collaborating Centre for Mental Health (2004) Eating Disorders: Core Interventions in the Treatment and Management of Anorexia Nervosa, Bulimia Nervosa and Related Eating Disorders. National Clinical Practice Guideline no. CG9. London: British Psychological Society \& Gaskell. 
Palmer, R. L. (1996) The management of anorexia nervosa. Advances in Psychiatric Treatment, 2, 61-68.

Palmer, R. L. (2000) Helping People with Eating Disorders: A Textbook for Clinicians. Chichester \& New York: John Wiley \& Sons.

Palmer, R. L. \& Treasure, J. (1999) Providing specialised services for anorexia nervosa. British Journal of Psychiatry, 175, 306-309.

Palmer, R. L., Birchall, H., Damani, S., et al (2003) A dialectica behavior therapy program for people with eating disorder and borderline personality disorder - description and outcome. International Journal of Eating Disorders, 33, 281-286.

Robinson, P. (2003) Day treatments. In The Essential Handbook of Eating Disorders (eds J. Treasure, U. Schmidt \& E. van Furth). Chichester: John Wiley \& Sons.

Royal College of Psychiatrists (2000) Eating Disorders in the UK: Policies for Service Development and Training (Council Report CR87). London: Royal College of Psychiatrists.

Royal College of Psychiatrists (2005) Guidelines for the Nutritional Management of Anorexia Nervosa (Council Report CR130). London: Royal College of Psychiatrists.

Russell, G. F., Szmukler, G. I., Dare, C., et al (1987) An evaluation of family therapy in anorexia nervosa and bulimia nervosa. Archives of General Psychiatry, 44, 1047-1056.

Sullivan, P. (1995) Mortality in anorexia nervosa. American Journal of Psychiatry, 152, 1073-1074.

Treasure, J. \& Bauer, B. (2005) Assessment and motivation. In The Essential Handbook of Eating Disorders (eds J. Treasure, U. Schmidt \& E. van Furth). Chichester: John Wiley \& Sons.

Wilson, T. \& Shafran, R. (2005) Eating disorders guideline from NICE. Lancet, 365, 79-81.

World Health Organization (1992) The ICD-10 Classification of Mental and Behavioral Disorders. Geneva: WHO.

\section{MCQs}

1 Children under 16 years of age with anorexia nervosa:

a should be offered treatment that involves their family

b respond better to conjoint family therapy than to family therapy in which they and their family are seen separately

c should have the opportunity of meeting with clinicians alone, without their family

d should receive family interventions that directly address the eating disorder

e should be advised to avoid their educational studies until after recovery.
2 Adults with anorexia nervosa:

a are characteristically ambivalent about seeking help

b rarely kill themselves

c should always receive a special form of cognitivebehavioural therapy

d need to have their physical health monitored throughout their treatment

e often have comorbid depression or depressive symptoms.

3 Anorexia nervosa:

a is a new disorder that came to prominence only in the 1960s

b almost never ends in recovery after more than 10 years of illness

c has a mortality rate that is among the highest for functional mental disorders

$\mathrm{d}$ is easily confused with hyperthyroidism

e has been the subject of more research trials than bulimia nervosa.

4 In treating anorexia nervosa:

a weight restoration is necessary but not sufficient for full recovery

b new atypical neuroleptic drugs have been shown to have a specific beneficial effect

c brief therapy - 20 sessions over 20 weeks - is the treatment of choice

d SSRIs are highly beneficial

e feeding cannot be considered a treatment in law.

5 In-patient treatment of anorexia nervosa:

a is recommended for most people

b is usually associated with clinically important weight gain

c is supported by a good evidence base

$\mathrm{d}$ is usually associated with a weight gain of about $2 \mathrm{~kg}$ a week

e needs to be conducted under legal compulsion in about half of cases. 